
internationales

vol. 20 - $n^{\circ} 2 \mid 2004$

L'asile politique en Europe depuis l'entre-deux-guerres

\title{
L'engagement comme ressource facilitant l'intégration. Le cas des réfugiés latino-américains en Suède
}

Frédéric Vabre

\author{
(2) OpenEdition \\ Journals \\ Édition électronique \\ URL : https://journals.openedition.org/remi/990 \\ DOI : $10.4000 /$ remi.990 \\ ISSN : $1777-5418$ \\ Éditeur \\ Université de Poitiers \\ Édition imprimée \\ Date de publication : 1 septembre 2004 \\ Pagination : 187-194 \\ ISBN : 2-911627-37-7 \\ ISSN : 0765-0752
}

Référence électronique

Frédéric Vabre, «L'engagement comme ressource facilitant l'intégration. Le cas des réfugiés latinoaméricains en Suède », Revue européenne des migrations internationales [En ligne], vol. 20 - n² 2 | 2004, mis en ligne le 25 septembre 2008, consulté le 16 avril 2022. URL : http://journals.openedition.org/ remi/990 ; DOI : https://doi.org/10.4000/remi.990

Ce document a été généré automatiquement le 16 avril 2022.

(c) Université de Poitiers 


\title{
L'engagement comme ressource facilitant l'intégration. Le cas des réfugiés latino-américains en Suède
}

\author{
Frédéric Vabre
}

1 Les sciences sociales s'interrogent souvent sur la manière de penser l'intégration. De plus en plus influencée par les politistes, la tendance actuelle de la réflexion européenne semble se dégager des seuls aspects sociaux et culturels pour réévaluer, à travers la notion de citoyenneté, la dimension politique de celle ci. L'intégration deviendrait ainsi définie comme le passage "du statut d'immigré à celui de citoyen ». (Rea, Tripier) C'est en partie dans cette démarche que nous souhaitons nous inscrire, à travers quelques éléments d'analyse du processus d'intégration des réfugiés latinoaméricains en Suède.

2 Souvent décrite comme un vieil État nation traditionnellement homogène sur le plan culturel et religieux (Rundblom, 1999), la patrie de Gunnar Myrdal a profondément changé de visage en quelques décennies, notamment du fait d'une politique relativement généreuse en matière de droits d'asile ${ }^{1}$. Au cour de l'année 2002, une enquête exploratoire a été menée dans le cadre d'un programme d'échange international par un groupe d'étudiants ${ }^{2}$ intéressés à plusieurs titres par l'insertion des réfugiés latino-américains, considérés généralement comme "éloignés culturellement » du groupe ethnique dominant. Cette étude s'est fondée sur une série de 25 entretiens approfondis menés auprès de migrants de la première génération ${ }^{3}$, habitant la ville de Växjö, au sud du pays ${ }^{4}$. L'un des constats majeurs qui ressort de cette étude sur la première génération d'immigrés concerne l'importance de la variable politique, que l'on évoque habituellement dans l'analyse de la deuxième génération (Rea, Tripier 2003). En effet, partisans à des degrés divers des mouvements de gauche latino-américains et pourchassés par les dictatures militaires dans les années soixantedix et quatre-vingt, les réfugiés du sous-continent ont d'abord bénéficié de leurs engagements au moment de leur réception. Ensuite, ils ont su utiliser leur goût pour l'engagement collectif comme une ressource leur permettant de s'insérer dans les 
structures de ce que certains persistent à appeler, pour mieux souligner son homogénéité, la « communauté suédoise » (Orfali, 1992).

\section{Un contexte d'arrivée favorable aux militants de la Démocratie}

\section{Une migration doublement « politique »}

Les mouvements migratoires constitués par le déplacement vers la Suède de milliers de latino-américains s'inscrivent parmi d'autres grandes vagues d'immigration ${ }^{5}$ des années soixante et soixante-dix, reflets des conflits internationaux et intra-étatiques. Cette migration latino-américaine revêt un double caractère politique, tant dans les raisons de départ comme dans celles de l'arrivée.

D'une part, les migrants fuyaient leurs pays en raison de leurs engagements. Il s'agissait de militants de partis de gauche, de militants syndicaux ou de sympathisants de ces mouvements, qui s'opposaient aux dictatures militaires. Ils étaient à l'époque dans des situations très difficiles: emprisonnés, guérilleros, recherchés par la police directement ou non (certains n'étaient que des proches d'opposants exposés). Une de nos enquêtés a, par exemple, dû se cacher dans une ambassade au Chili. Un homme raconte quant à lui comment sa famille a été expulsée d'Argentine. Un Chilien nous explique :

«J'ai rejoint un groupe politique chargé de défendre l'ancien régime. (...) J’ai été arrêté et mis en prison. Ma peine avait été fixée à 18 ans ferme ».

D'autre part, d'un point de vue interne à la Suède, le terme de " politique " migratoire n'a jamais été employé à meilleur escient. La décision d'accueillir les opposants latinoaméricains a été le fruit de la configuration politique suédoise du moment (Mella, 1990 ; Hadenius, 1999) Avant les coups d'état militaires, le gouvernement suédois dirigé par Olof Palme, portait un grand intérêt à la situation du Tiers monde ${ }^{6}$. Il soutenait explicitement les mouvements révolutionnaires de gauche d'Amérique latine, au point que le Chili est devenu rapidement un sujet majeur de la politique étrangère suédoise. Un des enquêtés nous affirme que le Premier Ministre suédois était perçu comme un ennemi des militaires, qui l'appelaient "Palme, le communiste». Après l'assassinat d'Allende, le Chili prit le relais du Viêt-nam comme victime symbolique et mobilisatrice de la nocivité de l'impérialisme américain. Les syndicats, dont l'influence sur les politiques migratoires est essentielle (Orfali, 1992), ont appuyé fortement la décision de recevoir en Suède les opposants aux juntes militaires. Ils ont ainsi contribué à renforcer la légitimité de leur présence, et du même coup à prévenir leur infériorisation en tant qu'immigrés. L'opinion favorable qui les attendait allait ainsi les aider dans leur installation (Lundberg, 1989).

6 Cette empathie de la plupart des Suédois est reconnue par les agents publics spécialisés sur les questions d'intégration que nous avons rencontrés. Les migrants en sont également très conscients. Ils connaissent tous l'investissement important des autorités, spécialement d'olof Palme, dans la possibilité de fuir leurs pays menaçants. La rhétorique employée est proche ici de l'idée de libération et de Salut, comme le montre ce Chilien : 
«Les gens dans les mouvements de résistance ont eu des contacts avec des organisations en Suède (...) C'est arrivé à la connaissance du Gouvernement suédois. Il a réussi à nous faire sortir et nous a offert de venir en Suède ".

7 Pour la plupart, la Suède était le seul pays à leur faire une telle faveur. Une Bolivienne exprime ainsi sa gratitude ${ }^{7}$ à son pays d'accueil :

«J'ai reçu plus de la Suède que de n'importe quel autre pays. Donc j'ai envie de lui donner le meilleur de moi même ».

8 Si l'on en croit les deux agents publics que nous avons interrogé à la municipalité et au bureau des migrations de la ville, les réfugiés latino-américains bénéficiaient ainsi de sérieux atouts pour s'insérer dans la société suédoise. La première vague de migrants de 1973 est notamment arrivée en Suède sans démarche de régularisation à accomplir. La deuxième vague de $1978 \mathrm{a}$ eu à peine plus de difficultés.

\section{Des politiques d'intégration ambitieuses}

Les réfugiés latino-américains arrivent à la charnière de deux époques. Les politiques traditionnelles à l'égard des migrants, assimilationnistes, sont en effet abandonnées. C'est en 1975, après une grande enquête nationale sur l'immigration, que l'on assiste à la consécration du paradigme multiculturaliste dans la loi. Cette nouvelle politique est résumée par le triptyque : "traitement égal », " participation» et «liberté de choix culturels » (Rundblom, 1998). Ce dernier objectif exprime l'idée que l'acquisition d'une identité culturelle suédoise devient facultative, la possibilité étant offerte de conserver son identité d'origine. L'objectif est de sortir de l'injonction paradoxale des politiques traditionnelles d'intégration : « on vous accepte, mais reniez vous » (Rea Tripier, 2003), au profit d'une logique de choix.

10 Pour la mettre en œuvre, la Suède mène une action d'envergure, qui passe par une administration répartie sur tout le territoire : une centaine de bureaux des migrations, qui vont encourager les municipalités à avoir des politiques d'intégration locales. La préservation des cultures d'origine est prioritaire. On met également en place un processus de soutien aux associations d'immigrés, qui sont subventionnées et qui participent à un conseil pour les questions relatives aux immigrés, rattaché au ministère du travail (Orfali, 1992)

11 Le contexte économique était à cette époque très favorable. Les réfugiés étaient ainsi pris en charge immédiatement: plans personnalisés comprenant cours de suédois et insertion professionnelle rapide. En dépit de la volonté de la majorité de repartir en Amérique latine ${ }^{8}$, qui a freiné l'apprentissage de la langue, leur qualification généralement haute et la structuration du marché du travail leur permettaient de s'insérer rapidement, sans même maîtriser le suédois. «Les temps étaient meilleurs. Plus de boulots, mieux payés» nous explique l'agent du bureau de l'intégration de la municipalité. Certes, il ne faudrait pas brosser un portrait trop idyllique de cette époque: les qualifications des réfugiés en particulier, n'ont pas été intégralement reconnues, comme nous l'explique ce Bolivien arrivé en 1981 :

«Les gens ont eu des boulots moins biens que là bas. Les médecins, par exemple, ils

les ont embauchés comme infirmiers, et les infirmiers comme aide-soignants ».

12 En dépit de ce déclassement, souvent temporaire, l'arrivée des réfugiés latinoaméricains en Suède a donc été marquée par une époque particulière, faite de facilités économiques et de bienveillances politiques. 


\section{Le goût de l'engagement collectif, clef vers l'intégration des latino-américains}

13 L'intérêt pour la politique, ou plutôt pour « le » politique, qui caractérise les migrants latino-américains, explique le retournement de leurs systèmes de souhaits. Alors qu'au départ l'envie de retourner défendre la démocratie dans leur pays dominait, l'impossibilité politique de traverser l'Atlantique, les déceptions liées aux «chocs de retour " (Gudykunst, Kim, 1997) l'acculturation et l'attachement à la société égalitaire suédoise les ont poussé à rester. Tout se passe comme si le désir d'engagement s'était finalement reporté sur le pays-hôte: le capital militant se trouve alors reconverti, réinvesti et sert de levier pour faciliter l'intégration.

\section{Des combats latino-américains aux engagements suédois}

Parmi les personnes que nous avons rencontrées, deux préoccupations politiques se dégagent assez nettement: la sauvegarde de l'état providence et la lutte contre l'extrême droite.

Les idéaux de justice sociale des opposants aux inégalités des sociétés latinoaméricaines ont trouvé en Suède un terrain d'application. L'égalitarisme, les politiques redistributives sont la concrétisation de ce que les migrants défendaient dans leurs pays. Aujourd'hui menacé par les politiques néolibérales en vogue, portées notamment par l'Union Européenne, le modèle suédois d'État providence trouve dans les réfugiés latino-américains ses défenseurs les plus acharnés. Une Chilienne s'en fait l'avocate :

«Je pense qu'en Suède nous voulons tous garder notre État providence. Même si beaucoup râlent à propos des impôts élevés, il faut le garder ».

La montée des mouvements d'extrême droite en Europe est un autre grand sujet d'inquiétude'. Une Chilienne nous fait ainsi part de ses craintes :

« Je pense que ce sera de plus en plus difficile pour les immigrés, à cause de la vague d'extrémisme de droite qui influence l'Europe aujourd'hui ».

17 Une Bolivienne est également sur ce registre de la peur :

« Pour moi, la Suède représente la justice sociale. Mais il y a des dangers contre ça avec la montée des mouvements racistes en Europe ».

\section{Les comportements électoraux}

« Je n'ai pas voté la dernière fois, car je ne suis pas satisfait des politiciens, (...) cette fois par contre, je vais y aller ». (un Chilien).

18 La question du vote n'est qu'un aspect de la citoyenneté, mais il est sans doute le plus symbolique. Dès 1975, les ressortissants étrangers établis en Suède ont eu le droit de vote aux élections locales, ce qui constitue une avancée majeure, peu copiée par les autres États européens ${ }^{10}$. La participation électorale des migrants est difficile à connaître. On estimait, au début des années quatre-vingt dix, entre $50 \%$ et $60 \%$ la part des étrangers participant aux scrutins (Orfali, 1992). Cependant, il faut garder à l'esprit que les acquisitions de nationalité sont très importantes, de l'ordre de $60 \%$, et qu'il est ensuite difficile pour les élections nationales de différencier les migrants naturalisés des Suédois issus du groupe ethnique majoritaire. En ce qui nous concerne, nous avons 
demandé aux enquêtés naturalisés s'ils comptaient voter aux élections législatives de septembre 2002. Tous, sans exception, ont répondu par l'affirmative ${ }^{11}$.

\section{La participation aux structures}

19 Engagés dans leur société d'accueil, tous nos interviewés participent à des organisations. Ces organisations sont bien sûr de plusieurs types.

D'abord, on peut distinguer les organisations spécifiques aux immigrés: association chilienne, bolivienne... Elles ont joué un rôle majeur dans les politiques d'intégration des immigrants (Orfali, 1992). L'État suédois les a rapidement institutionnalisées et subventionnées. L'argument explicité est de faire participer les étrangers aux décisions qui les concernaient; la raison latente renvoie sans doute plus à une logique classique de contrôle social. Ces associations ont pour but politique la représentation des minorités. Elles permettent également de perpétuer la culture du pays d'origine, par l'organisation d'activité de loisirs : musique, danse... aujourd'hui largement ouvertes à tous les Suédois. Mais si ces associations ont été très " englobantes ", nos interviewés participent à bien d'autres projets associatifs: clubs de sport locaux, associations humanitaires ou écologistes.

Plusieurs interviewés sont également membres d'un parti politique ${ }^{12}$, et considèrent cet engagement comme le signe le plus fort de leur appartenance à la société suédoise :

«Je fais partie de cette société, j'ai passé beaucoup de temps au sein du Parti social

démocrate (...) ça me donne le sentiment d'être chez moi ici. » (une Chilienne).

Une autre femme, venue de Bolivie et aujourd'hui suédoise, est membre active du Parti de la Gauche à Växjö ${ }^{13}$, et a été élue sur une liste aux municipales. Certains de nos interviewés ont également mentionné leur appartenance à un syndicat. L'un d'entre eux est même élu dans les organes dirigeants de la puissante centrale METALL.

Tous ces engagements ne renvoient pas néanmoins aux mêmes logiques. Adhérer à une association "communautaire " (chilienne ou autre) est une démarche située dans le temps, immédiatement postérieure à l'arrivée en Suède. C'est ensuite, généralement, lorsque le processus d'acculturation est bien engagé, que le réfugié va participer à une organisation détachée de cet enjeu. La nature des organisations auxquelles le migrant adhère est ainsi un bon moyen d'avoir un indice de son degré d'intégration politique Il y a une montée dans l'implication militante dans nombre de récits, lorsqu'on passe d'une participation à des groupes sportifs ou parentaux, à un investissement dans des groupes aux objectifs plus politisés. En outre, à l'intérieur de ceux-ci, les logiques d'élection ou le rôle de porte-parole n'impliquent pas le même engagement que la simple adhésion. Les évolutions qui nous sont contées témoignent en tous cas de cette progressive participation aux organisations suédoises.

24 En dépit des finalités différentes qu'ils poursuivent, la propension des migrants latinoaméricains à investir des structures correspond parfaitement à ce que les pouvoirs publics suédois attendent des immigrés. Les politiques d'intégration passent en effet par le rôle de l'institutionnel, rempart contre toute exclusion de la société suédoise. L'assimilation culturelle n'étant pas requise depuis 1975, s'insérer, en Suède, c'est intégrer les structures: école, autorités locales, milieu de travail, santé... qui fonctionnent toutes sur la base de cadres normatifs suédois. Au delà de ces structures classiques, toutes les sphères de la vie sociale sont institutionnalisées : "tout ce qui 
appartient au privé est transféré au public, parce que tout en Suède est collectif, public et contrôlable par la communauté dans son ensemble » (Orfali, 1992 : 189)

Ce modèle d'intégration par le goût du collectif transparaît dans la réponse d'une interviewée à la question : Comment définiriez-vous le mot "intégration»?

«S'intégrer... je crois que c'est deux choses: c'est participer, socialement,

politiquement, mais aussi être accepté comme citoyen et comme personne ».

Ces quelques observations nous conduisent à penser que la construction et la mise en récit de ce que nous appelons une "identité latino-nordique" comprennent une dimension d'engagement politique non négligeable, qui a structuré l'arrivée et l'acceptation des réfugiés latino-américains par le groupe ethnique dominant, et qui en outre a contribué à favoriser leur insertion dans les structures de la société suédoise.

\section{BIBLIOGRAPHIE}

ARBETSMARKNAD DEPARTMENT (1984) Latin amerikaner I Sverige- en översikt.

Regeringskansliets offsetcentral, Stockholm.

BAROU Jacques (1997) La Suède et l'intégration des femmes immigrées Migrations études nº 74, pp. $1-8$.

BOUJNAH Stéphane (2002) L'inoxydable modèle suédois, Cahiers En temps réel, $\mathrm{n}^{\circ}$ 6, décembre, téléchargeable sur httm://en.temps.reel.free.fr

DECKER Jennifer, KARLSSON Soraya, PETERSSON Maritha, VABRE Frédéric, (2002), The integration of Latin American immigrants in Växjö. An Overview étude de terrain réalisée sous la direction de Svante LUNDBERG à la Social Sciences School de l'Université de Växjö. (inédit)

GUDYKUNST (W.B.), KIM (Y.Y.), (1997) Communicating with strangers : an approach to Intercultural Communication, McGraw Hill, Boston.

HADENIUS Stig (1999) Swedish politics during the 20th century, Conflict and consensus, The Swedish Institute, Stockholm,

JEDERLUND Lars (1998) De la politique de l'immigration à la politique de l'intégration, Actualités suédoises $\mathrm{n}^{\circ} 422$.

LUNDBERG Svante (1989) Flyktingskap ; latinamrikansk exil i Sverige och Västeuropa, Tryck Studentlitteratur, Lund.

MELLA Orlando (1990) Chilenska Flyktingar i Sverige, CEIFO, Stockholm.

ORFALI Kristina (1992) Suède. De l'immigré au réfugié politique in Lapeyronnie Didier (dir.) Immigrés en Europe. Politiques locales d'intégration, La documentation française, Paris, pp. 165-191.

REA Andrea, TRIPIER Maryse (2003). Sociologie de l'immigration, La Découverte, Paris, 128 p.

REGERINGSKANSLIET (2002). Swedish integration policy for the 21st century, Rapport du gouvernement, Stockholm. 
RUNDBLOM Harald (1999). La Suède, société multiculturelle, articles publiés sur le site officiel

d'information sur la Suède : http://www.Sweden.se.

\section{NOTES}

1. Jusqu'aux années soixante-dix, la Suède connaît une immigration de travail, mais n'accueille pas beaucoup de réfugiés. La première loi adoptée par la Suède sur les étrangers, en 1914, limite le droit de séjour en Suède. C'est néanmoins ce texte qui introduit le droit d'asile. La loi dispose qu'un étranger ne peut être expulsé dans des circonstances «telles que le fait qu'il vient d'un pays éloigné, ou qu'il en a été banni, ou qu'il y serait vraisemblablement poursuivi ou condamné pour des délits politiques ». Cependant, ce nouveau droit est appliqué sans zèle. Malgré l'arrivée d'Hitler au pouvoir en 1933, la Suède accueille peu de réfugiés d'Allemagne. Jusqu'en 1942, la politique d'asile reste très restrictive. Ensuite, devant le nombre croissant de réfugiés, et pour donner des gages aux alliés après une neutralité suspecte, les autorités suédoises se décident enfin à accueillir les réfugiés qui le demandent. (Rundblom, 1998).

2. Nous étions quatre étudiants issus du Programme d'études interculturelles de l'Université de Växjö pour réaliser ce fieldwork, dirigés par Svante Lundberg (cf. références).

3. La sélection des interviewés s'est basée sur des critères d'âge, de sexe et de nationalité, afin de couvrir un éventail de situations aussi complet que possible.

4. Le choix de cette agglomération moyenne se justifie par l'existence, à proximité, de deux camps de réfugiés: Alvesta et Moheda. Ces derniers expliquent la présence de près de 1500 latino-américains (enfants issus d'au moins un parent migrant compris) dans cette commune de 75000 habitants.

5. Iraniens, Irakiens, Chiliens, Argentins, Péruviens, Kurdes, Érythréens...

6. Cette attention au Tiers monde caractérise traditionnellement le Parti social démocrate suédois. L'actuel Premier Ministre Göran Persson est le premier à ne pas l'avoir évoquée dans son discours de politique générale.

7. Il convient de rester prudent quant à la démonstration de cette gratitude. Des stratégies délibérées de présentation de soi ne sont pas à exclure.

8. Une Bolivienne: "Nous étions des réfugiés politiques, et nous avions en tête de retourner dans nos pays. Pour nous, la Suède était juste une parenthèse dans nos vies : plus ça serait rapide, mieux ça serait. La volonté de s'intégrer n'était pas très présente. Beaucoup de gens attendaient la première occasion pour partir... ".

9. Nos interviews, réalisées peu après le 21 avril 2002, qui a vu le Front National accéder au deuxième tour de l'élection présidentielle en France, sont marquées par cet événement, qui a fait la une de toute la presse suédoise.

10. La Suède et les Pays Bas sont les seuls pays de l'Union Européenne a avoir accordé aux étrangers le droit de vote aux élections locales, et ce malgré les multiples débats qui ont eu lieu ailleurs.

11. Il s'agissait bien d'intentions et non de votes effectifs.

12. Il faut tout de même rappeler ici que les partis politiques suédois attirent beaucoup plus de militants qu'en France. Le parti social démocrate compte par exemple 152000 membres, alors que le parti socialiste français n'atteint même pas ce chiffre dans un pays six fois plus peuplé. Cette situation serait justement due à l'ouverture forte qui est pratiquée vis à vis des jeunes et des personnes issues de l'immigration. (Boujnah, 2002).

13. Il s'agit du parti le plus à gauche du spectre politique suédois. À Växjö, il est l'allié du parti social démocrate et des verts dans la majorité municipale. 
INDEX

Mots-clés : insertion sociale, réfugiés, réseaux communautaires

Index géographique : Suède

\section{AUTEUR}

\section{FRÉDÉRIC VABRE}

Étudiant en DEA Action publique et sociétés contemporaines, École Normale Supérieure de Cachan, 61, avenue du Président Wilson, 94235 Cachan Cedex. E-mail : fvabre12@yahoo.fr. 